Sharif University of Technology
Scientia Iranica
SCIENTIA

\title{
Ferrofluid appendages: Ferrofluid vortex container - A numerical investigation into free surface shape and vortex flow in ferrofluids for different relative densities
}

\author{
M.A. Feizi Chekab and P. Ghadimi* \\ Department of Marine Technology, Amirkabir University of Technology, Hafez Ave, No 424, Tehran, P.O. Box 15875-4413, Iran.
}

Received 3 April 2016; received in revised form 27 September 2016; accepted 31 October 2016

KEYWORDS
Ferrofluid;
Density;
Shape;
Vortex;
Numerical simulation.

\section{Introduction}

Knowing and controlling are among the most essential motivations of engineers in many fields to continue engineering human surroundings through their knowledge and art of control for optimizing the reciprocal relation of mankind and their environment. Controlling fluid flows, among other control issues, has always been one of the most critical problems for which the main solution has mostly been the use of an overwhelming mass of solids that are structured in the form of reservoirs,

\footnotetext{
*. Corresponding author. Tel.: +98 $216454311 \%$;

Fax: +982166412495

E-mail address: pghadimi@aut.ac.ir (P. Ghadimi)
}

\begin{abstract}
The use of ferrofluids as shapeable external appendage to a submerged body and as a mean of vortex flow induction is studied in this paper. Ansys-CFX ( of the surrounding non-magnetic fluids. It is demonstrated that the height, width, and curvature of ferrofluid can be controlled by magnet size and strength. It is also observed that ferrofluid mass loss may occur due to gravity, which should be addressed in designing a fluid appendage. Subsequently, vortex production inside the ferrofluid is investigated a shear flow on the magnet. It is shown that ferrofluid can contain complex vortices of these parace

(C) 2017 Sharif University of Technology. All rights reserved.
\end{abstract}

pipes, valves, propellers, etc. To reduce the need of solid state for fluid control, smart fluids were born.

Ferrofluids, among others, have changed the engineering view of the flow control. Nowadays, ferrofluids are used in heat transfer [1], optics [2-4], micropumps and microfluidic devices [5,6], electro-magnetic control of the droplet [7-9], nanotechnologies [10,11], and many other industrial fields [12].

Ferrofluid has also been welcomed in the electronic art sector, where it has been used for producing ferrofluid sculptures [13] and ferrofluid displays and interfaces [14,15], which have made the application of these fluids public. Examples of these artistic applications are displayed in Figure 1.

Resler and Rosenweig [17] in 1964 and Shliomis [18] in 1968 presented governing equations of the ferrofluids motion, called ferro-hydrodynamics equations. 


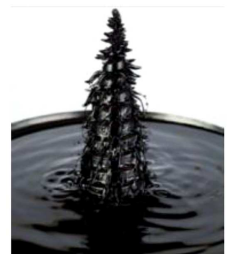

(a)

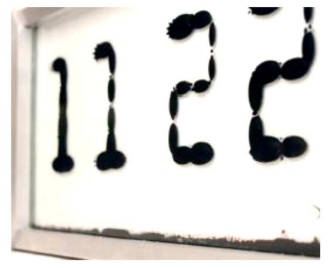

(b)

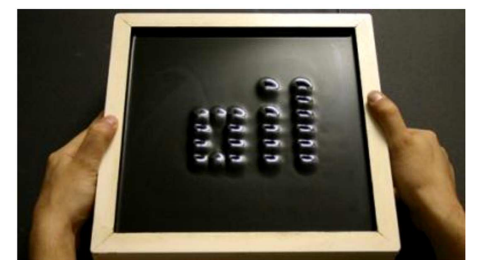

(c)

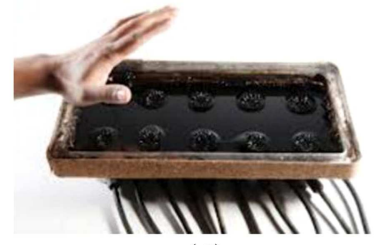

(d)

Figure 1. (a) Fluid sculptures [13], (b,c) ferrofluid display [14,15], and (d) ferrofluid interface [16].

Many researchers have experimentally investigated Ferrohydrodynamics. Shliomis and Raikher [19] and Cowley and Rosnweing [20] focused on the free surface stability of the ferrofluids. Hejazian and Nguyen [21] also investigated the behavior of non-magnetic particles suspended in ferrofluids. Different aspects of ferrofluid flows have also been studied, analytically and numerically. Shliomis et al. [22,23] focused on improving the equations of ferrohydrodynamics and ferrofluids magnetization and relaxation behavior. Lavrora [24] presented numerical methods for predicting static axisymmetric shapes of ferrofluids. Rabbia et al. [25] also numerically studied the heat transfer of ferrofluids in a lid-driven cavity.

As pointed out earlier, in addition to heat transfer and magnetic sealing and suspension, shaping ferrofluids are used in many fields where shape of the fluid is of high importance, mainly in visualization and electronic arts. Accordingly, the basic idea behind the current study is to investigate the possibility of using the smart fluids and, particularly, ferrofluids as external shape changing appendages for the submerged bodies in water. Examples of this application may include "fluid fins" and "fluid tails" in bionics, "fluid propellers" for marine vessels, or even "fluid structures" for designing shape changing controllable bodies as a marine vessel, for the enhanced applications. Another important goal of the present study is to assess the feasibility of generating complex flows inside the fluid appendages in order to add surface flow control to the "fluid structures". For instance, the focus of the present paper is firstly to investigate the free surface shape of ferrofluids in a secondary non-magnetic fluid medium with a different density under the influence of permanent magnets with different sizes and field strengths. Secondly, the possibility of vortex generation inside the ferrofluid and using the ferrofluid as a vortex container is investigated.

Vortex control around moving bodies in air or water can highly affect the pressure distribution, velocity field, and, hence, the efficiency of the body in motion. However, actively producing and controlling vortices is not always simple in most mediums including air and water. The present study numerically shows the possibility of producing stationary ferrofluid vortices on a submerged wall. A schematic of the problem is illustrated in Figure 2.
In the next section, the governing equations, which are numerically solved by Ansys-CFX software, are presented. Afterwards, the ability of the software in simulating ferrohydrodynamics is assessed by modeling the stable state of a ferrofluid around a vertical electric current. Subsequently, the main investigation is presented, which was previously outlined.

\section{Governing equation}

As mentioned earlier, the numerical simulations are conducted using the Ansys-CFX solver. In the present section, the governing equations applied in the numerical processes in the mentioned solver are briefly presented [26]. The continuity of mass and momentum is as follows:

$$
\begin{aligned}
& \frac{\partial \rho}{\partial t}+\nabla(\rho U)=0 \\
& \frac{\partial \rho U}{\partial t}+\nabla \cdot(\rho U \otimes U)=-\nabla p+\nabla \cdot \tau+F_{e \mathrm{mag}} .
\end{aligned}
$$

Here, $U$ is the fluid velocity vector and $t, p, \tau$, and $\rho$ are time, pressure, viscous stress, and fluid density, respectively. Also, $F_{\text {emag }}$ represents the momentum due to electromagnetic forces. Because of high complexity of the problem, this term is evaluated using the Maxwell's equations as follows:

$$
\begin{aligned}
& \nabla \times E=-\frac{\partial B}{\partial t}, \\
& \nabla \times H=J+\frac{\partial D}{\partial t}, \\
& \nabla \cdot B=0 \\
& \nabla \cdot D=\rho_{e},
\end{aligned}
$$

where $B, D, E, H, J$, and $\rho e$ are magnetic induction, electric displacement, electric field, magnetic field, current density, and electric charge density, respectively. Using these equations, $F_{\text {emag }}$ is extracted to be:

$$
F_{\text {emag }}=\mu_{0} M \nabla H \text {. }
$$

In this equation, $M$ is fluid magnetization, $H$ is the magnetic field, and $\mu_{0}$ is the magnetic permeability of the vacuum. The presented momentum source assumes that free surface is stable. In other words, the 


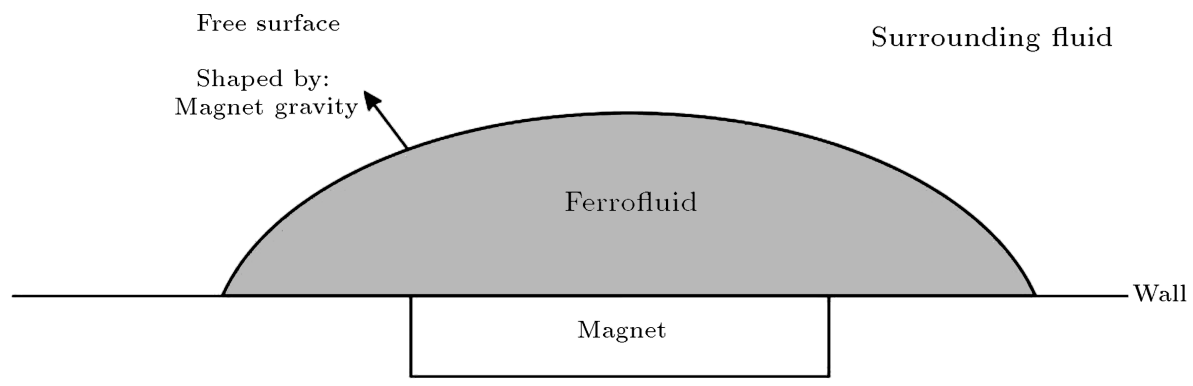

(a)

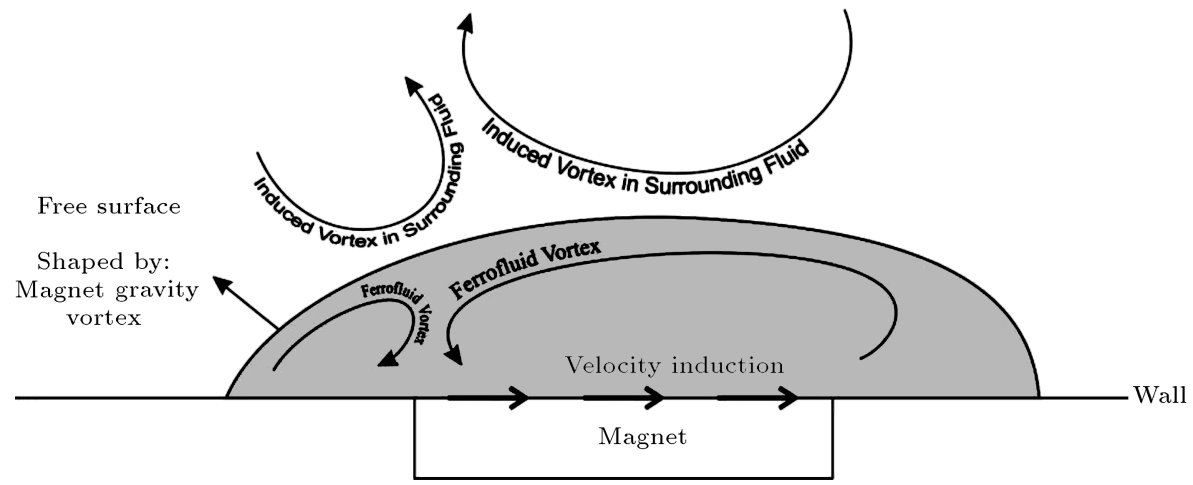

(b)

Figure 2. Schematic of the ferrofluid vortex container and its effect on the surrounding fluid: (a) without vortex, and (b) with induced vortex.

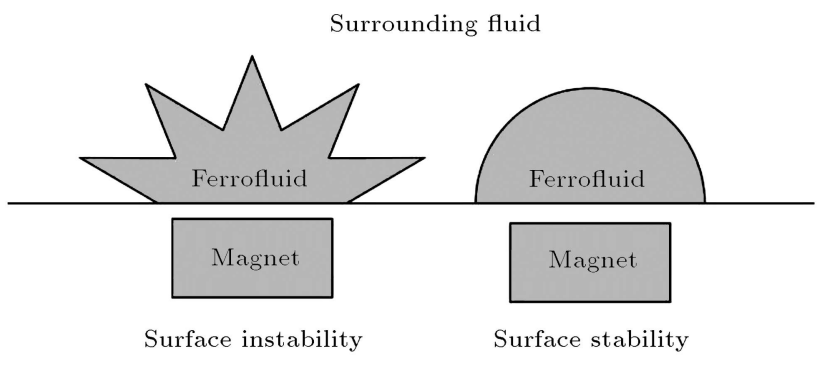

Figure 3. Stable and instable free surfaces in ferrofluids.

surrounding fluid, the ferrofluid, and the magnetic field are set in a way that no instability occurs on the free surface. The differences between stable and unstable free surfaces are displayed in Figure 3.

The focus of the present study is on the stable free surface, where no spikes occur. Finite Volume method is used by Ansys-CFX for solving the presented governing equations for ferrohydrodynamics problems. Validation of the ferrohydrodynamics software is presented in the following section.

\section{Validation}

Prior to the numerical investigation of the main problem, it is necessary to assess the validity of the solver. To this end, two different problems have been considered to validate both the free surface and the flow of the ferrofluid in magnetic fields.

\subsection{Free surface validation}

To validate the modeling of free surface response of the ferrofluid to magnetic fields, the ascension of a ferrofluid along a vertical electric current carrying rod is simulated and the obtained result is compared with the analytical solution. Due to the electric current inside the rod, a magnetic field is created around the rod, which attracts the ferrofluid and ascends along the rod and reaches an equilibrium state due to the gravitational force. This is better illustrated in Figure 4, which shows this experiment by Rosenweig [27].

As observed in Figure 4, the ferrofluid is stretching along the rod due to the electric current magnetic

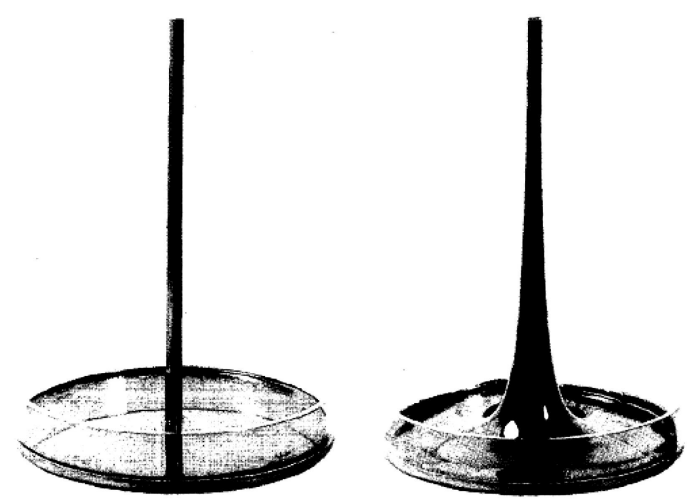

Figure 4. Response of a magnetic fluid to the steady electric current passing through a vertical rode [27]. 


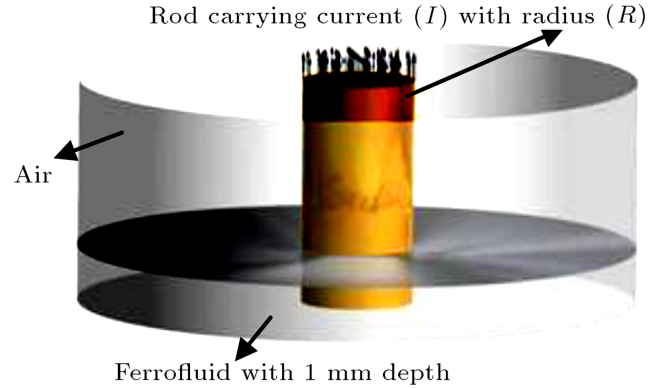

Figure 5. Problem setup for the validation problem.

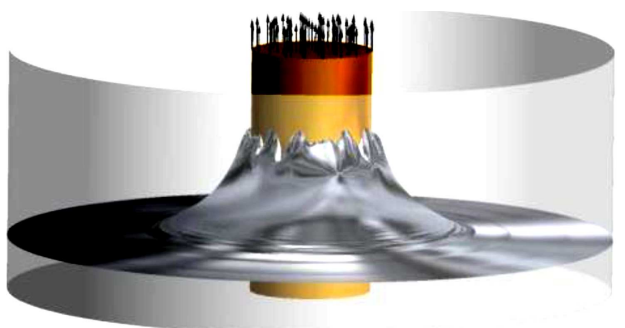

Figure 6. Free surface elevation obtained for test case number 1.

field. The analytical solution to the stable elevation of the ferrofluid is presented [27] by:

$$
\Delta h=\frac{\mu_{0} \chi I^{2}}{8 \pi^{2} \rho g R^{2}} .
$$

In this equation, $\Delta h$ is the elevation of the ferrofluid, $\mu_{0}$ is the magnetic permeability of the vacuum, $\chi$ is the fluid magnetic sensitivity, $I$ is the electric current, and $\rho, g$, and $R$ are the ferrofluid density, the gravitational acceleration, and the rod radius, respectively.

The problem setup is shown in Figure 5.

In the present simulation, the current is assumed to be $3.5 \mathrm{~A}$, the rod radius is set at $1 \mathrm{~mm}$, the ferrofluid density is $997 \mathrm{~kg} / \mathrm{m}^{3}$, and the magnetic sensitivity varies as presented in Table 1 along with the results obtained for each case.

As evident in Table 1, the elevation obtained for the three considered cases is very close to the analytical solution and the error of simulation ranges from $1.21 \%$ to $2.29 \%$, which is considered acceptable for most of the engineering simulations. The computed ferrofluid free surface for test case 1 is displayed in Figure 6 .

\subsection{Magnetic flow validation}

To validate the flow simulation of ferrofluids in magnetic fields, the Hartman problem [28,29] is considered here. The Hartman problem consists of the flow of a magnetic fluid in a channel, in the presence of magnetic field. For this problem, the Hartman number should be defined as:

$$
\mathrm{Ha}=B d \sqrt{\frac{\zeta}{\rho \nu}},
$$

where $B$ is the magnetic field strength and $\rho, \nu, \zeta$, and $d$ are the density, kinematic viscosity, electrical conductivity of the fluid, and channel width, respectively. The Hartman problem is solved for $\mathrm{Ha}=0,3.2,6.3$, 9.5 , and 15.8 in this article and results are compared with analytical solutions. The obtained results of the analyses are presented in Figure 7 .

As observed in Figure 7, the Root Mean Square of Errors (RMSE) of the simulation compared to analytical solutions is $0.16 \%$ for all cases. More details on this validation are presented in a previous work by the current authors [30].

After the validation of the solver, the numerical study of ferrofluids with different relative densities and under the influence of different magnets is presented in the next section.

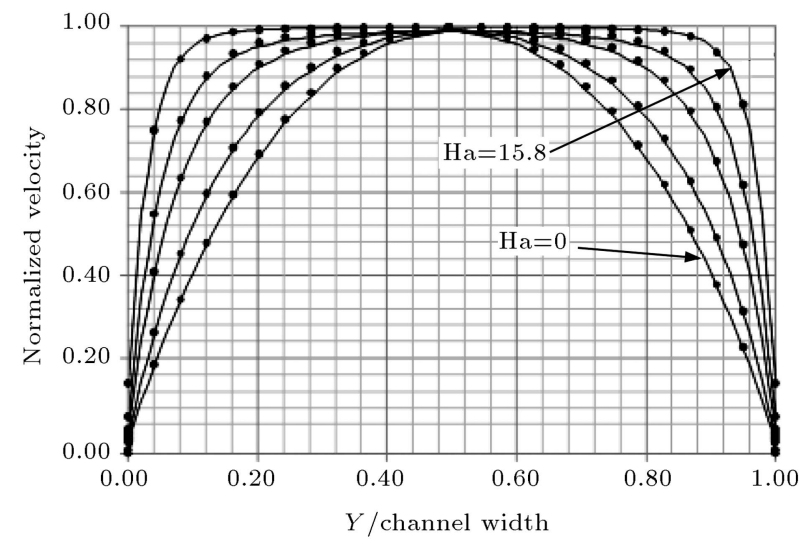

Figure 7. Normalized velocity vs. normalized vertical coordinate at different Hartman numbers for numerical $(-)$ and analytical $(\bullet)$ solutions.

Table 1. Input values and results obtained for the validation of the software in different cases.

\begin{tabular}{ccccc}
\hline Test \# & $\boldsymbol{X}$ & $\begin{array}{c}\boldsymbol{d h} \\
\text { analytical } \\
(\mathbf{m m})\end{array}$ & $\begin{array}{c}\boldsymbol{d h} \\
\text { numerical } \\
(\mathbf{m m})\end{array}$ & Error \\
\hline 1 & 50 & 0.986944293 & 1.0059 & $1.92 \%$ \\
2 & 75 & 1.480416439 & 1.4625 & $1.21 \%$ \\
3 & 100 & 1.973888586 & 2.019 & $2.29 \%$ \\
\hline
\end{tabular}

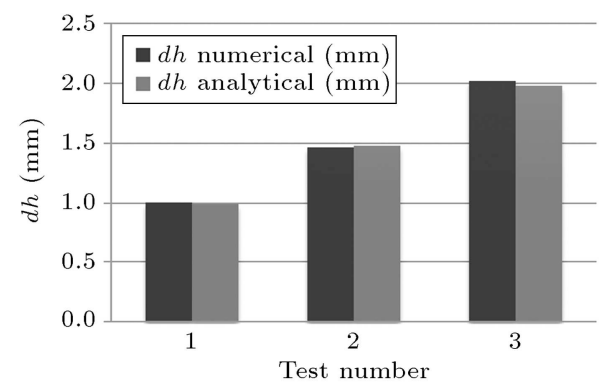




\section{Results and discussion: Ferrofluids free surface shape}

As pointed out earlier, the main goal of the present study is to numerically analyze free surface shape of a ferrofluid in surrounding fluids with different densities. Additionally, the assessment of the feasibility of using a ferrofluid as a flow control device via actively producing vortices inside the ferrofluid is of concern. In the present section, the first part of the study is presented, where the ferrofluid is analyzed in different magnetic fields $\left(B_{\text {mag }}\right)$, magnet sizes $\left(L_{\text {mag }}\right)$, relative densities $s=\rho_{\text {ferrofluid }} / \rho_{\text {surrounding fluid }}$, and on horizontal and vertical walls (which is applied by altering the gravity direction). The setup of the considered problem is presented in Figure 8.

As shown in Figure 8, a very thin rectangular domain is selected with a magnet of variable length $\left(L_{\mathrm{mag}}\right)$. Also, an induced velocity on the magnet is assumed, which is set to zero in this part of the study, where no flow is necessary. Values of different considered variables are presented in Table 2 .

In the following subsections, ferrofluid on a horizontal wall is investigated and, then, the same study is carried out for the ferrofluid on a vertical wall to show the feasibility of the use of ferrofluid on moving objects which can rotate in any direction.

\subsection{Horizontal wall}

As previously mentioned, shape of the ferrofluid on a horizontal wall is analyzed in this subsection. The free surface shape for different relative densities for $L_{\text {mag }}=$ $1 \mathrm{~cm}$ and $B_{\text {mag }}=0.25 \mathrm{~T}$ along with magnetic field lines as a sample of the results are displayed in Figure 9.

As observed in Figure 9, the ferrofluid is agglomerated above the magnet. For higher relative densities $(s>1)$, the ferrofluid takes a bell form. However, for

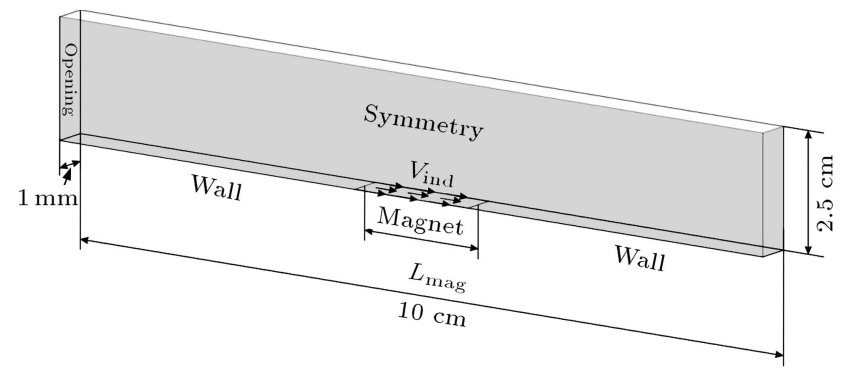

Figure 8. Setup of the conducted simulations.

Table 2. Simulation cases for the ferrofluid shape.

\begin{tabular}{ccccccccc}
\hline Wall & Horizontal & Vertical & & & & & \\
$\boldsymbol{L}_{\text {mag }}$ & $5 \mathrm{~mm}$ & $10 \mathrm{~mm}$ & & & & & \\
$\boldsymbol{B}_{\text {mag }}$ & $0.25 \mathrm{~T}$ & $0.5 \mathrm{~T}$ & & & & & \\
$\boldsymbol{s}$ & 0.5 & 0.75 & 0.9 & 1 & 1.1 & 1.25 & 1.5 \\
$\boldsymbol{V}_{\text {ind }}$ & $0.00 \mathrm{~m} / \mathrm{s}$ & & & & & & \\
\hline
\end{tabular}

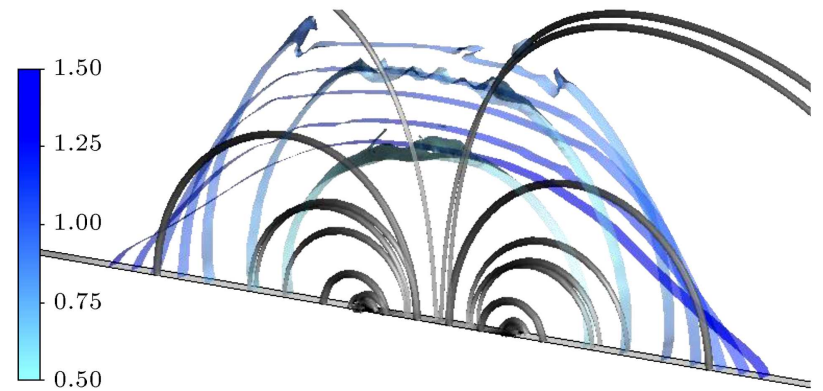

Figure 9. Free surface and magnetic field lines for $L_{\mathrm{mag}}=1 \mathrm{~cm}$ and $B_{\mathrm{mag}}=0.25 \mathrm{~T}$ at different densities $(s=0.5$ to 1.5$)$.

lower relative densities, the ferrofluid tends to ascend due to its positive buoyancy. This positive buoyancy causes the free surface shape to be more spherical. Also, a mass loss occurs due to the lack of magnetic force for retaining all the ferrofluid. Consequently, lesser relative density causes more ferrofluid loss, and thus smaller ferrofluid accumulation.

An equation used in the present manuscript is a dimensionless number, $B g$, to show the significance of gravitational versus magnetic forces as body forces acting on the fluids, as in:

$$
B g=\frac{\rho g L \mu}{B^{2}},
$$

where $\mu$ is the permeability of the magnetic fluid and $L$ is the characteristic length of the problem, which is the height of the ferrofluid surface from its bottom line. Higher $B g$ numbers indicate higher effects of gravity compared to magnetic field on the fluid surface. The range of $B g$ number in the present study is between 0.0014 and 0.011 , which implies the smallness of gravity compared to the magnetic forces.

Also, the Bond number (Bo) is hereby used to show the ratio between the surface tension and body forces, which is defined as:

$$
\mathrm{Bo}=\frac{B^{2} L}{\sigma \mu},
$$

where $\sigma$ is the surface tension and other parameters have been defined before. It should be noted that because of very low $B g$ numbers in this study, the main body force is due to magnetic field rather than the gravity; therefore, Eq. (11) is written in terms of the magnetic field. High Bo means negligible effect of the surface tension compared to the body forces, which is hereby the magnetic force. The Bo number in the present study ranges between $6.5 \mathrm{e} 4$ and $7.5 \mathrm{e} 6$, which are very high bond numbers, showing the very small effect of the surface tension compared to the body forces.

To better observe and compare the results for all the 36 considered cases, the free surfaces are plotted together for $s \geq 1$ in Figure 10 . 

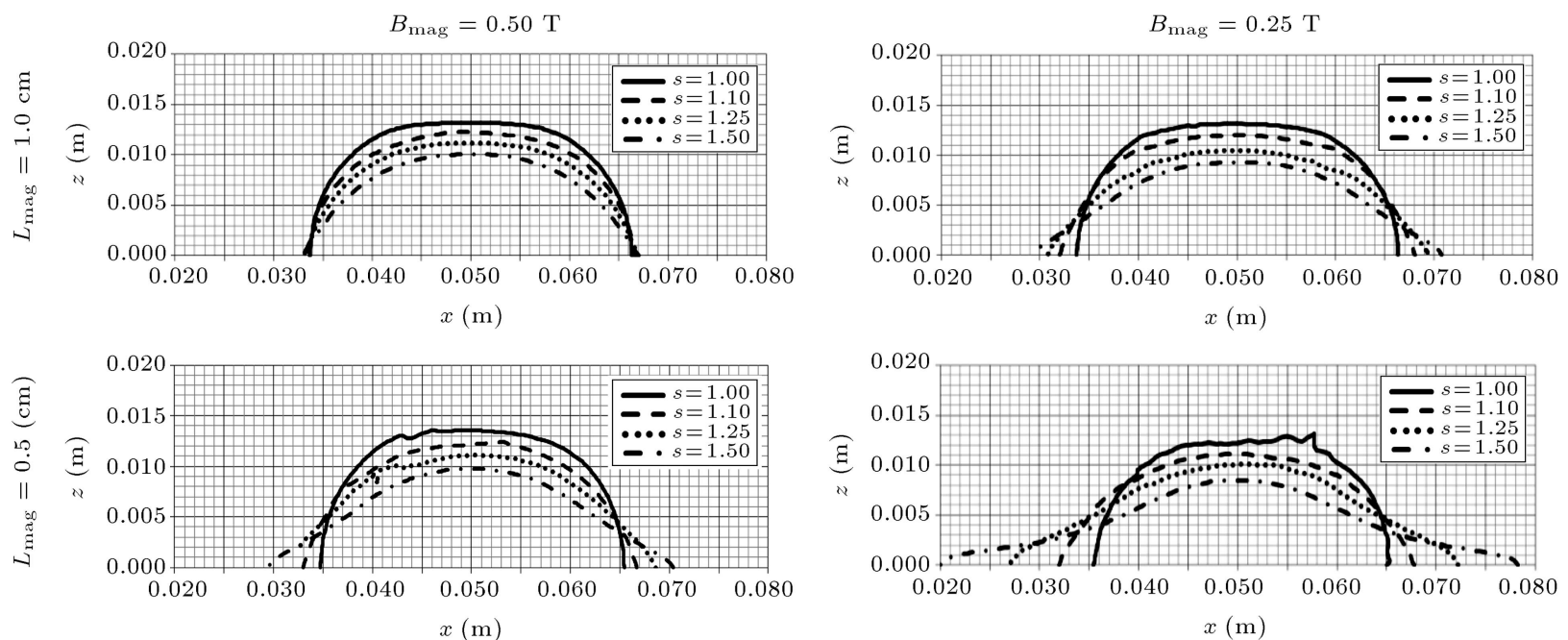

Figure 10. Ferrofluid shapes for $s \geq 1$ for different values of $B_{\text {mag }}$ and $L_{\text {mag }}$, with $B g$ equal to 0.0017 up to 0.01 and Bo from $4 \mathrm{e} 5$ to $3 \mathrm{e} 6$.
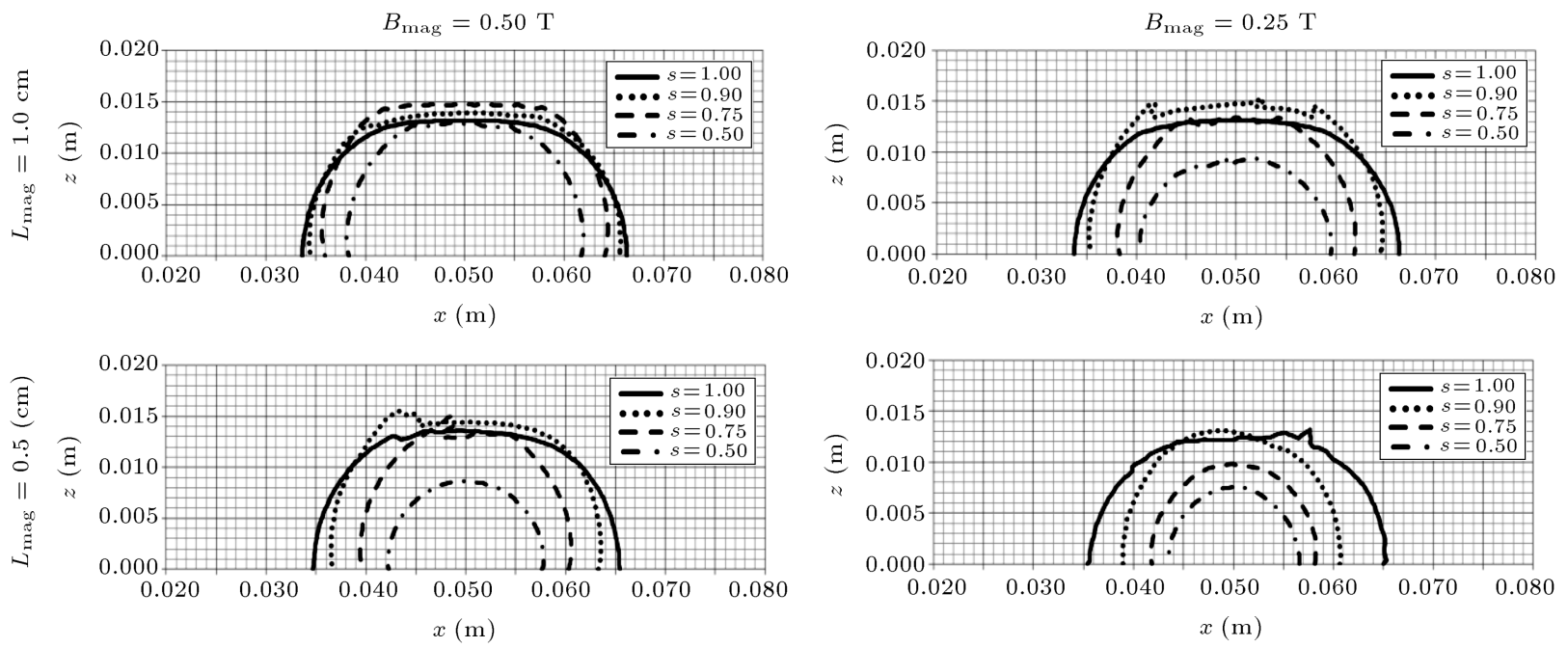

Figure 11. Ferrofluid shapes for $s \leq 1$ for different values of $B_{\text {mag }}$ and $L_{\text {mag. }}$.

As evident in Figure 10, for $s=1$, there is no significant difference in the shapes of the ferrofluid for different magnet strengths and sizes. However, weaker magnet increases width of the ferrofluid and decreases the height of the ferrofluid for $s>1$, changing the shape of the ferrofluid from a nearly elliptical shape to a bell shape. Also, the smaller magnet increases the curvature change in the ferrofluid and causes a more concentrated elevation in the ferrofluid with higher relative densities. By calculating the $B g$ number using Eq. (10), it can be demonstrated that $B g$ number is between 0.0017 to 0.01 , which shows the very high effect of the magnetic field compared to the gravity. Ferrofluid shapes for $s \leq 1$ and for different values of $B_{\mathrm{mag}}$ and $L_{\mathrm{mag}}$ are also presented in Figure 11.

As observed in Figure 11, for $s<1$, a lower relative density increases the height of the ferrofluid and decreases the width of the base of the ferrofluid. However, more ferrofluid mass is lost, as relative density is decreased for a constant $B_{\text {mag }}$ and $L_{\text {mag }}$. Also, smaller magnet size increases these effects and produces more concentrated shapes. On the other hand, an increase in the magnet strength yields in a more stable ferrofluid shape for different fluids.

The above results indicate that it would be very hard to change the shape of a ferrofluid with the same density as the surrounding fluid by changing the specifications of a single magnet, because gravity does not participate in the shaping of the fluid and the magnetic field will result in a very similar shape for all cases. This could be an advantage in many cases and a disadvantage in many others. However, it is surely a sign of the fact that vast varieties of shapes and behavioral designs could be presumed as an external appendage to a submerged body. 


\subsection{Vertical wall}

Another focal point of the present study is the effect of transverse gravity. In Figure 11, the ferrofluid is shown to be agglomerated on a vertical wall.

As evident in Figure 12, the existence of transverse gravity highly changes the behavior of the ferrofluid for relative densities other than unity. Also, the ferrofluid mass loss occurs this time for both $s<1$ and $s>1$. As observed, for stronger and larger magnets, these effects are diminished. It should be pointed out here that for an appendage which is subject to change of direction relative to the gravity direction, $s=1$ seems to be the best choice. Again, it is shown through the calculation of the $B g$ number that the gravitational energy is much lower than the magnetic energy.
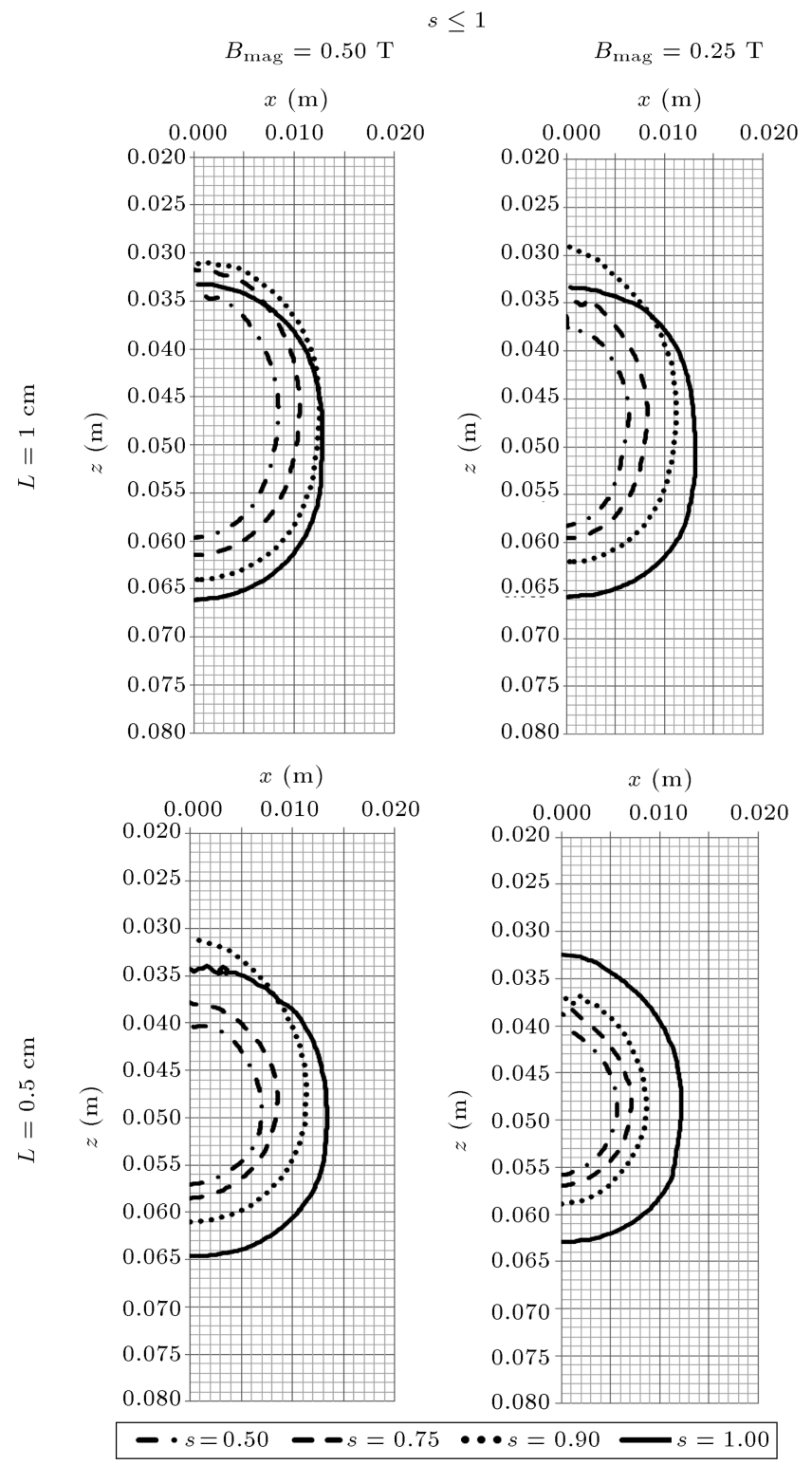

Figure 12. Ferrofluid shapes for different values of $s, B_{\text {mag }}$, and $L_{\text {mag }}$ on a vertical wall with $B g$ being equal to 0.0014 up to 0.011 and Bo number from 2.5e5 to $2.54 \mathrm{e} 6$.

\subsection{Surface tension effects}

Although the present paper focuses on the use of magnetic field and gravity in the shape control of a fluid with constant properties, it should be emphasized that the behavior of the ferrofluid could change by changing the fluid properties. As a brief demonstration of this fact, the surface tension coefficient between the ferrofluid and the surrounding fluid has been set to $0.0001 \mathrm{~N} / \mathrm{m}, 0.01 \mathrm{~N} / \mathrm{m}$, and $0.025 \mathrm{~N} / \mathrm{m}$ for $B=0.25 \mathrm{~T}$ and $s=1.25$. Figure 13 illustrates the shape of free surface with different surface tensions.

As observed in Figure 13 and as expected, increasing the surface tension slightly decreases the length of the base line of the ferrofluid and slightly increases the height of the ferrofluid. It is also shown in

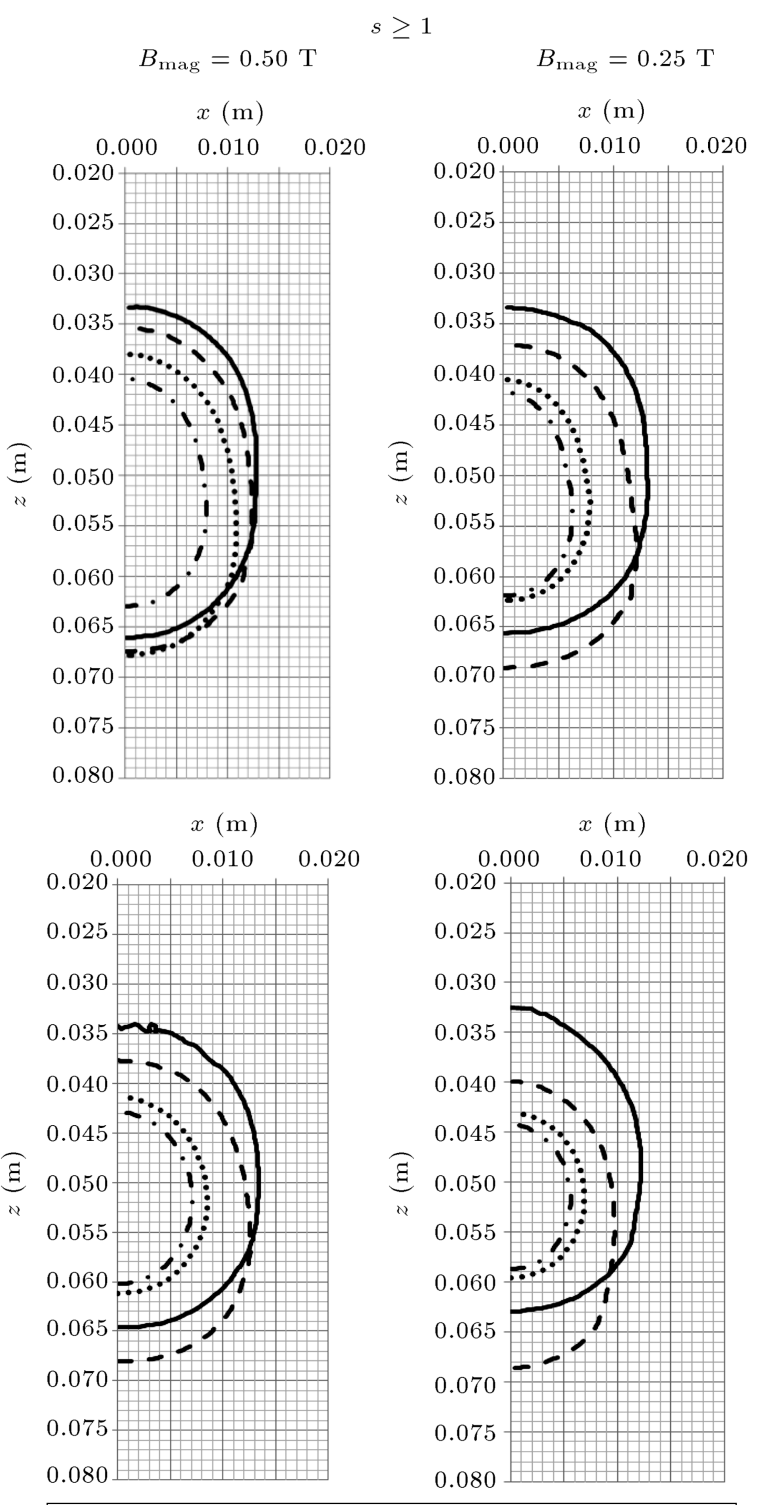




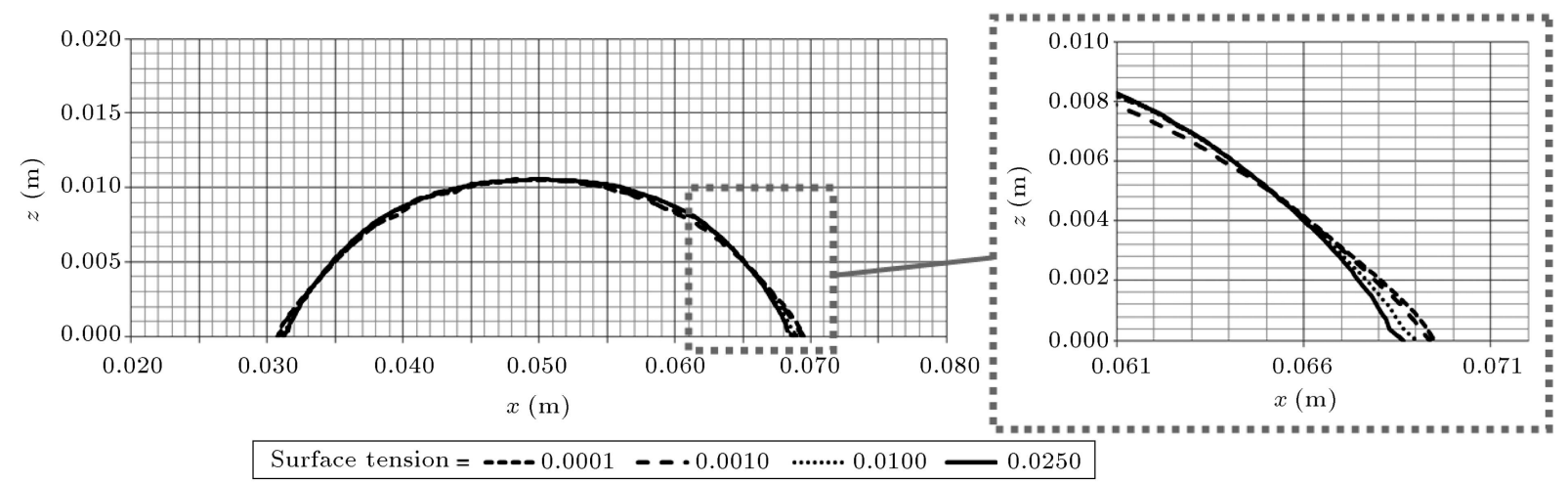

Figure 13. The ferrofluid shape in different surface tensions for $B=0.25 \mathrm{~T}, s=1.25$, and magnet length $=1 \mathrm{~cm}$.

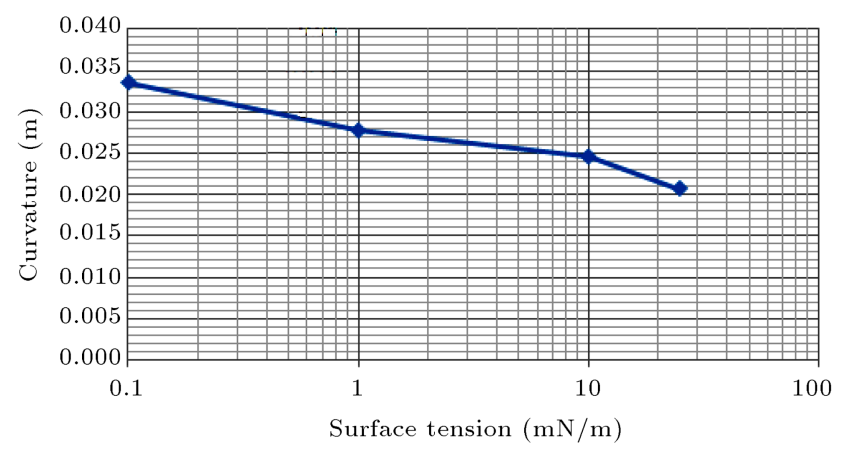

Figure 14. Curvature of the ferrofluid versus surface tension.

Figure 14 that increasing the surface tension decreases the curvature of the ferrofluid. In other words, surface tension slightly decreases the effect of the magnetic and gravitational forces on the equilibrium shape of the fluid.

\section{Results and discussion: Ferrofluid vortex}

\subsection{Effect of magnetic field and density ratio}

As mentioned in the introduction, after assessing the shape of the ferrofluid in different circumstances, it is important to assess the possibility of inducing fluid flow in the surrounding fluid via vortex production inside the ferrofluid. To this end, in the present section, $V_{\text {ind }}$ is set to be $0.05 \mathrm{~m} / \mathrm{s}$ (equivalent to $R e=500$ ) in the hope of producing vortices inside the ferrofluid. It is important to emphasize the fact that the ferrofluid is sustained on its place by a magnet on the wall of the body. In this section, the effects of magnet strength and relative density are also assessed on the vortex production inside the ferrofluid. The values of different parameters are displayed in Table 3.

Figure 15 illustrates the flow inside and around the ferrofluid for $B_{\mathrm{mag}}=0.25$ and $s=1.5$ as a sample result.

In Figure 15, vectors represent the velocity field and are accompanied by partial streamlines for better
Table 3. Simulation cases for ferrofluid vortices.

\begin{tabular}{cccccc}
\hline Wall & Horizontal & & & & \\
$\boldsymbol{L}_{\text {mag }}$ & $10 \mathrm{~mm}$ & & & & \\
$\boldsymbol{B}_{\text {mag }}$ & $0.25 \mathrm{~T}$ & $0.5 \mathrm{~T}$ & & & \\
$\boldsymbol{s}$ & 0.5 & 0.9 & 1 & 1.25 & 1.5 \\
$\boldsymbol{V}_{\text {ind }}$ & $0.05 \mathrm{~m} / \mathrm{s}(\operatorname{Re}=500)$ & & \\
\hline
\end{tabular}

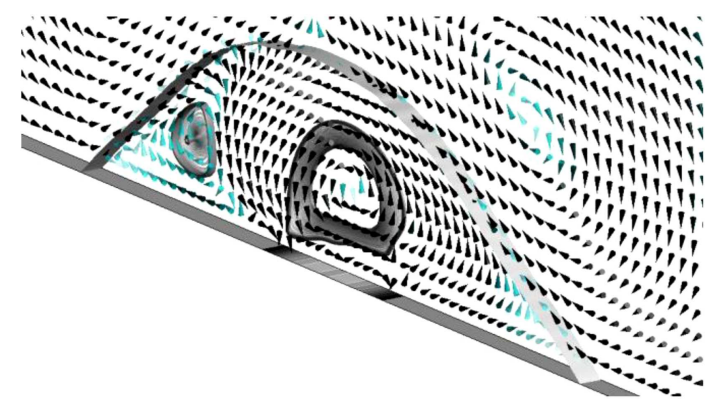

Figure 15. Ferrofluid shape and fluid flow inside and around the ferrofluid for $B_{\mathrm{mag}}=0.25$ and $s=1.5$.

showing the vortex cores created by the shear velocity $V_{\text {ind }}$. It is clearly observed that two main vortices are produced inside the ferrofluid due to the shear velocity on the magnet. Also, a velocity field is transferred to the surrounding fluid from the free surface of the ferrofluid. Similar to previous cases, Figure 16 illustrates the ferrofluid shapes after the vortex induction in different circumstances.

Also, to better visualize the velocity field and vortex cores, each case has been illustrated separately in Figure 17.

As evident in Figure 17, the presence of $V_{\text {ind }}$ causes at least two distinct vortices inside the ferrofluid; one main vortex, which is consistent with $V_{\text {ind }}$, and one smaller, which flows in the reverse direction. It is also observed that the flow inside the ferrofluid affects the flow in the surrounding fluid, which signals the possibility of external flow control via ferrofluid appendages. It is further observed that flow inside 

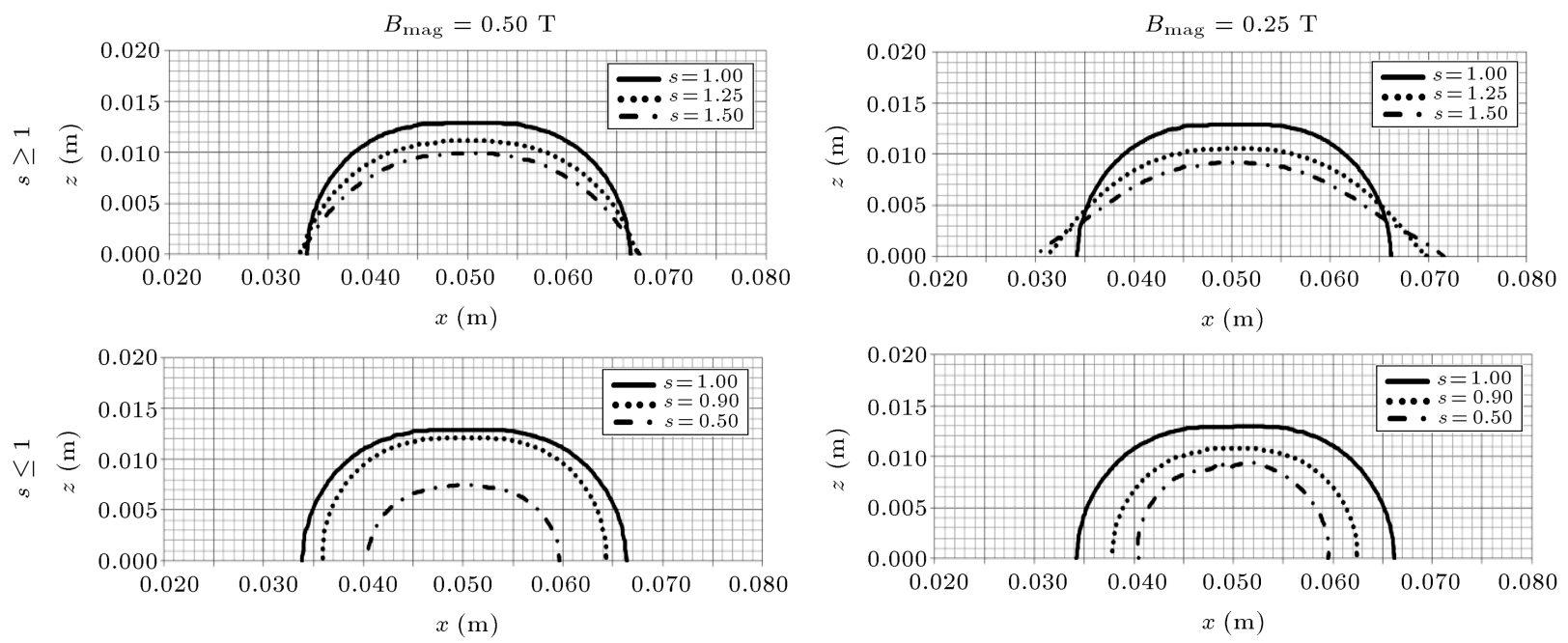

Figure 16. Ferrofluid shapes for different values of $s$ and $B_{\text {mag }}$ for ferrofluids with vortex.
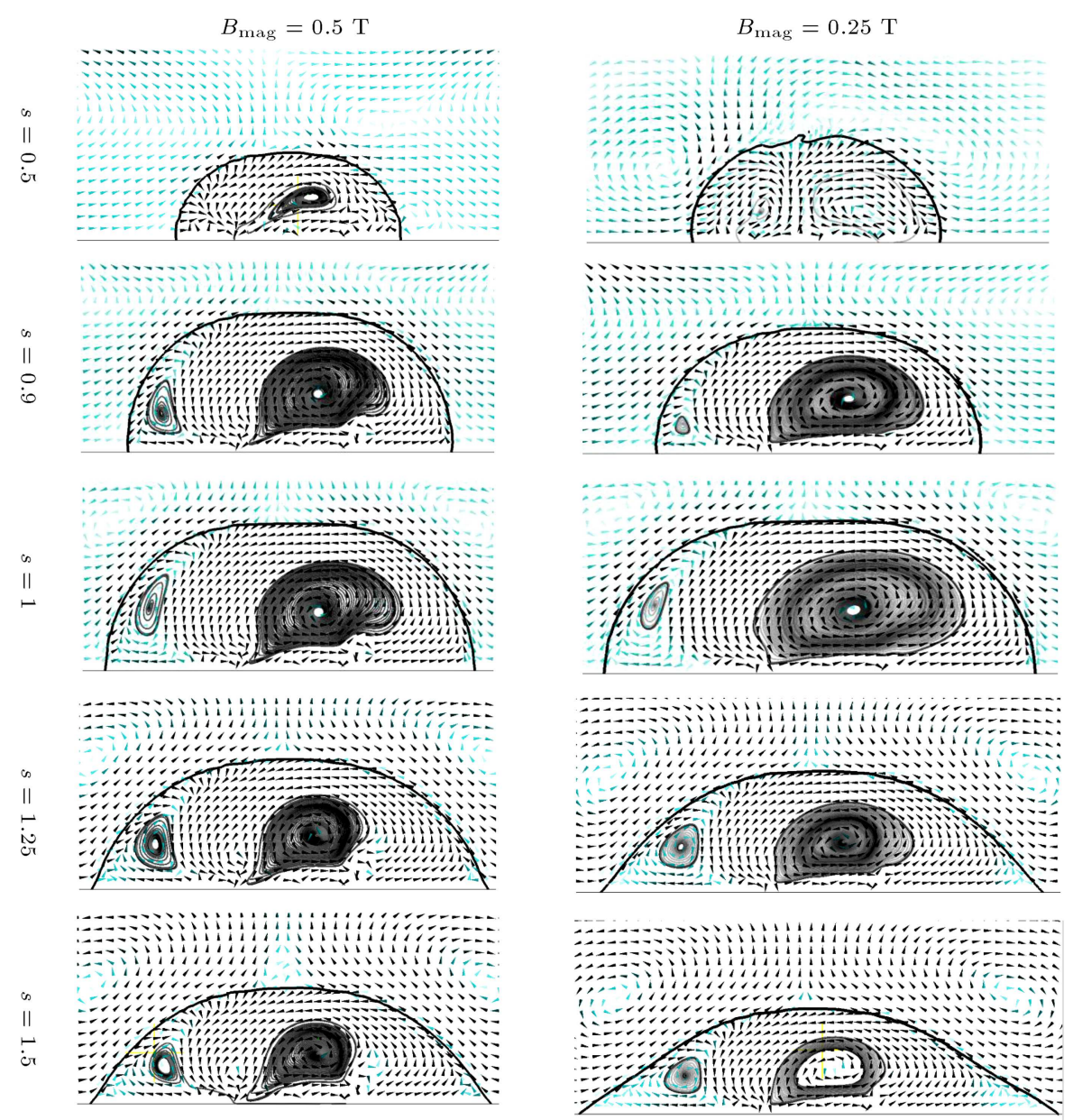

Figure 17. Ferrofluid shapes, velocity fields, and partial streamlines for different values of $s$ and $B_{\text {mag. }}$.

the ferrofluid affects the shape of the ferrofluid and reduces the curvature change of the free surface at higher relative densities. Therefore, it is possible to refine the shape of the ferrofluid by means of controlling the velocity field of the ferrofluid.

\subsection{Effects of Reynolds number}

Although the Reynolds number of the vortex container is not the main subject of the present study, its effects on the flow and shape of the vortex container are assessed here by altering the viscosity of the ferrofluid. 


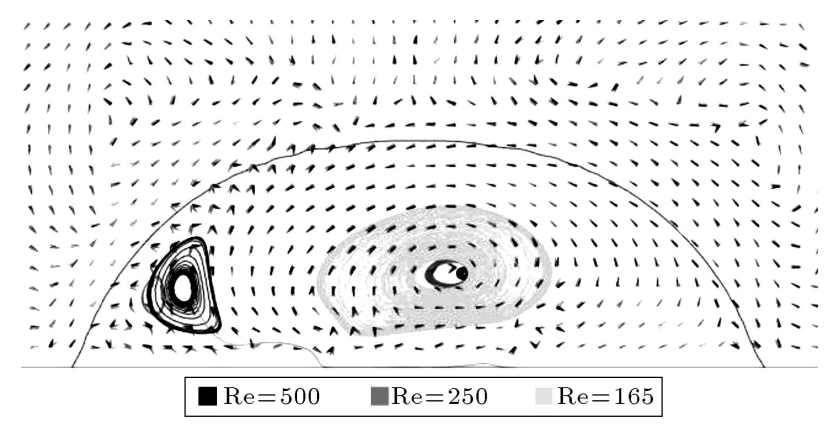

Figure 18. Velocity field and streamlines for different Reynolds numbers.

In these cases, the length of the magnet is $1 \mathrm{~cm}, B=$ $0.25 \mathrm{~T}, s=1.25$, and surface tension is considered to be $0.0001 \mathrm{~N} / \mathrm{m}$. The analysis is conducted for $\operatorname{Re}=165$, 250, and 500. Figure 18 displays the vortex container streamlines for different Reynolds numbers (different viscosities).

As observed in Figue 18, lower Reynolds numbers (higher viscosities) displace the main vortex core to the middle of the container. Also, the secondary vortex core is strengthened by increasing the Reynolds number. It is shown in Figure 18 that the secondary vortex vanishes for $\operatorname{Re}=160$. As shown in Figure 18, the Reynolds number highly affects the flow inside the vortex container and may be the subject of future studies on the ferrofluid appendages.

\section{Conclusions}

Nowadays, smart fluids, including ferrofluids, are growingly being used in different aspects of human life. The use of these fluids in optics, microfluidics, heat transfer, electronic arts, and many other fields has been proposed and even generalized for nearly half a century. However, many aspects of these fluids have not been explored yet. The current paper has focused on the possibility of using ferrofluids as an external appendage to create fluid vortices on a body submerged in a secondary non-magnetic fluid, like water, in which case a "fluid appendage" could be designed with ferrofluids and magnetic sources to serve as a flow control device. Accordingly, the present study has been conducted in two phases. The first phase has concentrated on the analysis of the ferrofluid free surface shape at different surrounding fluid densities on horizontal and vertical walls and under the influence of different magnet sizes and strengths, which resulted in 72 cases. The second phase of the present study has focused on the feasibility of producing vortices inside the ferrofluid in hope of influencing the flow of the surrounding fluid. This has been achieved by analyzing the ferrofluid at different surrounding fluid densities and different magnet strengths.

For conducting the numerical analysis, the finite volume solver Ansys-CFX has been validated using the analytical solution to ferrofluid static shape around an electric current carrying rod for validation of ferrofluid free surface, which led to errors less than $2.3 \%$. This is while the Hartman problem has been used to validate the ferrofluid flow in a magnetic field, which led to error of $0.16 \%$.

It has been demonstrated that height, width, and curvature of the ferrofluid can be controlled by the size and strength of the magnets. Moreover, ferrofluid mass loss may occur due to low relative density, low magnet strength, low magnet size, and the orientation of the ferrofluid wall relative to gravity. It has also been shown that vortices can be created inside the ferrofluid, which can, on the one hand, affect the flow of the surrounding fluid and, on the other hand, apply fine adjustments to the curvature of the ferrofluid surface, if necessary. Also, the effects of surface tension and viscosity have been roughly analyzed to show the importance of these parameters for future works.

The overall conclusion of the present study is the fact that use of ferrofluids as external shapeable appendages and vortex container seems possible but very complex, and needs thorough experimental studies.

\section{Nomenclature}

$\begin{array}{ll}\Delta h & \text { Elevation of the ferrofluid } \\ B & \text { Magnetic induction } \\ B g & \begin{array}{l}\text { Gravitational forces versus magnetic } \\ \text { force ratio }\end{array} \\ B_{\text {mag }} & \text { Magnetic field of the magnet } \\ \text { Bo } & \text { Bond number } \\ D & \text { Electric displacement } \\ E & \text { Electric field } \\ F_{\text {mag }} & \text { Electromagnetic forces } \\ g & \text { Gravitational acceleration } \\ H & \text { Magnetic field } \\ \text { Ha } & \text { Hartman number } \\ I & \text { Electric current } \\ J & \text { Current density } \\ L_{\text {mag }} & \text { Magnet length } \\ M & \text { Fluid magnetization } \\ p & \text { Pressure } \\ R & \text { Rod radius } \\ R e & \text { Reynolds number } \\ R M S E & \text { Root Mean Square of Errors } \\ s & \text { Relative density } \\ t & \text { Time } \\ U & \text { Fluid velocity vector }\end{array}$




\section{Greek letters}

$\begin{array}{ll}\tau & \text { Viscous stress } \\ \rho & \text { Fluid density } \\ \rho e & \text { Electric charge density } \\ \mu_{0} & \text { Magnetic permeability } \\ \zeta & \text { Electrical conductivity }\end{array}$

\section{References}

1. Lajvardi, M., Moghimi-Rad, J., Hadi, I. et al. "Experimental investigation for enhanced ferrofluid heat transfer under magnetic field effect", Journal of Magnetism and Magnetic Materials, 322(21), pp. 35083513 (2010).

2. Sheikholeslami, M. and Rashidi, M.M. "Ferrofluid heat transfer treatment in the presence of variable magnetic field", The European Physical Journal, 130, Article ID 115 (2015).

3. Khan, W.A., Khan, Z.H. and Haq, R.U. "Flow and heat transfer of ferrofluids over a flat plate with uniform heat flux", The European Physical Journal, 130(86), pp. 2198-2203 (2015).

4. Laird, P.R., Bergamasco, R., Berub, V. et al. "Ferrofluid based deformable mirrors: a new approach to adaptive optics using liquid mirrors", Proceedings of SPIE - The International Society for Optical Engineering, 4839(2) (2002).

5. Hartshorne, H., Backhouse, C.J. and Lee W.E. "Ferrofluid-based microchip pump and valve", Sens Actuators, 99, pp. 592-600 (2004).

6. Link, D.R., Grasland-Mongrain, E., Duri, A. et al. "Electric control of droplets in microfluidic devices", Angew Chem. Int. Ed., 45(16), pp. 2556-2560 (2006).

7. Long, Z., Shetty, A.M., Solomon, M.J. and Larson, R.G. "Fundamentals of magnet-actuated droplet manipulation on an open hydrophobic surface", Lab Chip, 9(11), pp. 1567-1575 (2009).

8. Nguyen, N.T., Ng, K.M. and Huang, X. "Manipulation of ferrofluid droplets using planar coils", Appl. Phys. Lett., 89(5), 052509 (2006).

9. Afkhami, S., Renardy, Y., Renardy, M. et al. "Fieldinduced motion of ferrofluid droplets through immiscible viscous media", J. Fluid Mech., 610, pp. 363-380 (2008).

10. Yellen, B.B., Fridman, G. and Friedman, G. "Ferrofluid lithography", Nanotechnology, 15, pp. S562S565 (2004).

11. Zahn, M. "Magnetic fluid and nanoparticle applications to nanotechnology", J. Nanopart. Res., 3, pp. 73-78 (2001).

12. Rosensweig, R.E., Ferrohydrodynamics, Cambridge University Press, London (1985).

13. Kodama, S. "Dynamic ferrofluid sculpture: Organic shape-changing art forms", Communications of The ACM, 51(6), pp. 79-81 (2008).
14. Koelman, Z., de Graaf, M.J. and Lee, H.J. "From meaning to liquid matters", Proceedings of the 21st International Symposium on Electronic Art (2015).

15. Frey, M. "SnOil - A physical display based on ferrofluid", http://www.freymartin.de/en/projects/ snoil, accessed on 2016 .

16. Koh, J., Karunanayaka, K., Sepulveda, J. et al. "Liquid interface: A malleable transient direct-touch interface", Proceedings of the rth International Conference on Advances in Computer Entertainment Technology, Taipei, Taiwan (2010).

17. Resler, E.L. Jr. and Rosensweig, R.E. "Magnetocaloric power", AIA A J., 2(8), pp. 1418-2 (1964).

18. Shliomis, M.I. "Equations of motions of a fluid with hydromagnetic properties", Soviet Physics JETP, 26, p. 665 (1968).

19. Shliomis, M.I. and Raikher, Y.L. "Experimental investigations of magnetic fluids", IEEE Transactions on Magnetics, MAG-16, 237 (1980).

20. Cowley, M.D. and Rosnweig, R.E. "The interfacial stability of a ferromagnetic fluid", J. Fluid Mech., 30(4), pp. 671-688 (1967).

21. Hejazian, M. and Nguyen, N.T. "Negative magnetophoresis in diluted ferrofluid flow", Lab Chip, 15, pp. 2998-3005 (2015).

22. Shliomis, M.I., Lyubimova, T.P. and Lyubimov, D.V. "Ferrohydrodynamics: an essay on the progress of ideas", Chem. Eng. Com., 67(1), pp. 275-290 (1988).

23. Shliomis, M.I. "Comment on Magnetoviscosity and relaxation in ferrofluids", Physical Review E, 64, Article ID: 063501 (2001).

24. Lavrova, O. "Numerical methods for axisymmetric equilibrium magnetic fluid shapes", Dissertation, Otto-von-Guericke-Universität Magdeburg (2006).

25. Rabbia, K.Md., Sahaa, S., Mojumdera, S. et al. "Numerical investigation of pure mixed convection in a ferrofluid-filled lid-driven cavity for different heater configurations", Alexandria Engineering Journal, 55(1), pp. 127-139 (2016).

26. Ansys CFX-Solver Theory Guide, Release 15.0, ANSYS, Inc (2015),

27. Rosensweig, R.E. "Magnetic fluids", Ann. Rev. Fluid Mech., 19, pp. 437-461 (1987).

28. DePuy, T.R. "Fluid dynamics and heat transfer in a Hartmann flow", BSc Thesis, Faculty of Rensselaer Polytechnic Institute, US (2010).

29. Thess, A., Krasnov, D., Boeck, T., Zienicke, E., Zikanov, O., Moresco, P. and Alboussiere, T. "Transition to turbulence in the Hartmann boundary layer", GAMM-Mitteileungen, 30, pp. 125-132 (2007). 
30. Feizi Chekab, M.A. and Ghadimi, P. "Taguchi parametric analysis of the effects of electrode and magnetic actuator characteristics on Lorentz forces and heat transfer of a weak low-profile magneto-hydrodynamic blanket propulsion system", Proceedings of the Institution of Mechanical Engineers, Part C: Journal of Mechanical Engineering Science, 231(19), pp. 35533568 (2017).

\section{Biographies}

Mohammad A. Feizi Chekab received his BSc and MSc degrees in Marine Engineering and Naval Architecture from Amirkabir University of Technology in years 2006 and 2009, respectively. He was then admitted to the $\mathrm{PhD}$ program in the Department of Marine Technology in year 2010 and is currently working on his dissertation. His main research interests include hydrodynamics and MHD and he has authored several articles on these topics.

Parviz Ghadimi received his $\mathrm{PhD}$ degree in Mechanical Engineering in 1994 from Duke University, USA. He served for one year as a Research Assistant Professor in the Mechanical Engineering Department and for six years as a Visiting Assistant Professor in Mathematics Department at Duke. He then joined the Department of Marine Technology at Amirkabir University of Technology, Iran, in Fall 2005. He is currently a Full Professor of Hydromechanics in this department. His main research interests include hydrodynamics, hydroacoustics, thermo-hydrodynamics, and CFD, and he has authored over one hundred scientific papers in these fields. 